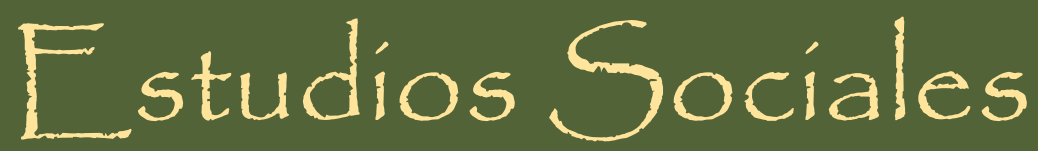

Revista de Alimentación Contemporánea y Desarrollo Regional Volumen 29, Número 54. Julio - Diciembre 2019 Revista Electrónica. ISSN: 2395-9169

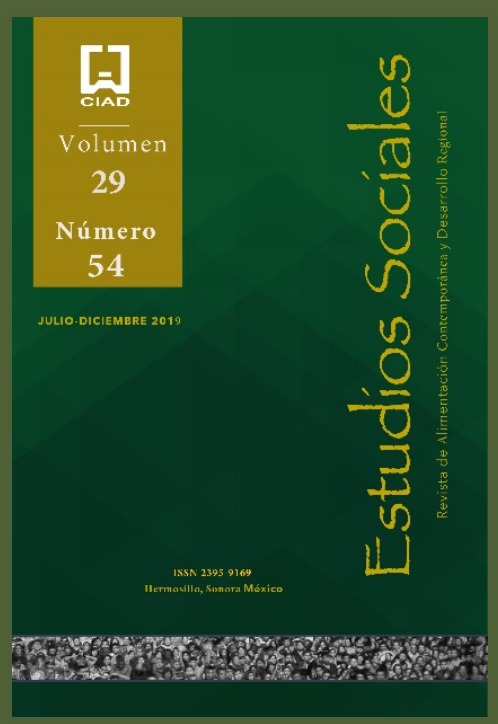

Percepción de la calidad y confianza en el jamón ibérico.

Perspectivas del consumidor y del vendedor

Perception of quality

and confidence in Iberian ham.

Consumer and seller perspectives

DOI: https://dx.doi.org/10.24836/es.v29i54.797

Pll: e19797

Laura Patricia Sánchez-Vega*:

https://orcid.org/0000-0003-2224-6207

Santiago Amaya-Corchuelo**:

https://orcid.org/0000-0001-6633-3842

Angélica Espinoza-Ortega*:

https://orcid.org/0000-0002-5968-0587

Fecha de recepción: 28 de marzo de 2019.

Fecha de envío a evaluación: 24 de abril de 2019.

Fecha de aceptación: 13 de mayo de 2019.

*Universidad Autónoma del Estado de México. Instituto de Ciencias Agropecuarias y Rurales.

Autora para correspondencia: Angélica Espinoza-Ortega. Doctorado en Ciencias Agropecuarias y Recursos Naturales.

Instituto Literario N0. 100. Col. Centro.

Toluca, estado de México, 50000.

Tel. 7222965552.

Dirección: laura.sanchez.pt@gmail.com

**Universidad de Cádiz, España.

Centro de Investigación en Alimentación y Desarrollo, A. C. Hermosillo, Sonora, México. 


\section{PERCEPCIÓN DE LA CALIDAD Y CONFIANZA EN EL JAMÓN IBÉRICO. PERSPECTIVAS DEL CONSUMIDOR Y DEL VENDEDOR \\ SÁnchez-Vega, Amaya-Corchuelo, Espinoza-OrTega}

\section{Resumen / Abstract}

Objetivo: Analizar las percepciones sobre la calidad de jamón ibérico a través de las apreciaciones de los consumidores y de los vendedores. Basándose en la confianza entendida como interacción entre ambos agentes de la cadena alimentaria. Metodología: Se utilizó una metodología cualitativa exploratoria aplicando un cuestionario a cien consumidores y treinta vendedores de jamón. Resultados: Se encontró que los actuales procesos de producción y la incorporación de tecnología al sector del jamón ibérico muestra cuatro tipos de percepciones que se clasificaron en actuales positivos, mejor en el pasado, neutrales y permanentes. Limitaciones: Fueron dos, la primera es que no se hizo una clasificación del tipo de vendedor; la segunda fue que el tamaño de la muestra no permite hacer generalizaciones, por lo cual para futuras investigaciones será necesario atender estas sugerencias. Conclusiones: Los cambios en la concepción de calidad se relacionan con la percepción sobre la aplicación de las normativas del sector del jamón, pero fundamentalmente dependen de las relaciones de confianza dinámicas y construidas entre consumidor y vendedor.

Palabras clave: alimentación contemporánea; calidad percibida; subjetividades; puntos de vista; construcción de confianza; atributos de crédito.
Objective: The aim is to analyze the perceptions about the quality of Iberian ham through the appreciations of consumers and sellers. Based on trust understood as interaction between both agents in the food chain. Methodology: A qualitative exploratory methodology was used, applying a questionnaire to 100 consumers and 30 ham vendors. Results: It was found that the current production processes and the incorporation of technology to the Iberian ham sector show four types of perceptions that were classified as positive current, better in the past, neutral and permanent. Limitations: There was no classification of the type of seller, and the size of the sample does not allow generalizations, so for future research it will be necessary to address these suggestions. Conclusions: The changes in the conception of quality are related to the perception on the application of the regulations of the ham sector, but fundamentally depend on the dynamic and built trust relationships between the consumer and the seller.

Key words: contemporary food; perceived quality; subjectivities; points of view; trust building; credit attributes. 


\section{Introducción}

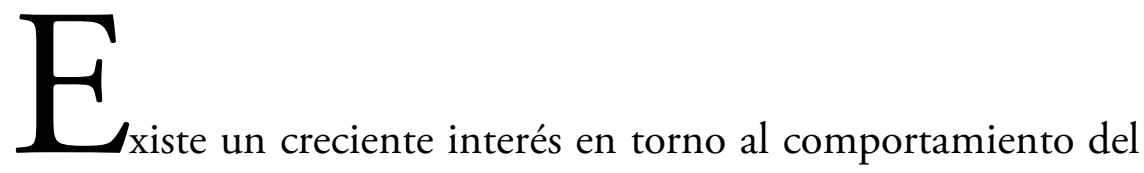
consumidor al decantarse por unos productos en detrimento de otros, sobre qué considera calidad y cómo la aplica en dichas elecciones. La calidad en los alimentos se ha estudiado, principalmente, desde la perspectiva de los productores, sin embargo, en las últimas décadas se ha puesto énfasis en conocer la perspectiva de los consumidores sobre distintas categorías de productos como los denominados tradicionales (Guerrero et al., 2010), orgánicos (Escobar-López, Espinoza-Ortega, Vizcarra-Bordi y Thomé-Ortiz, 2017), transgénicos (Sánchez y Barrena, 2004), los que tienen algún sello de calidad alimentaria (Amaya-Corchuelo, Sánchez-Vega, Aguilar y Espinoza-Ortega, 2016), entre otros.

Con el objetivo de tener una mayor comprensión del acto alimentario se intenta conocer los motivos de consumo (Espinoza-Ortega et al.2016), disposición a pagar el sobreprecio por cierto valor ańadido (Jaramillo, 2015), expectativas, creencias, percepciones (Morales, Guerrero, Claret, Guàrdia y Gou, 2008), así como entender las señales intrínsecas (sabor, olor, color, textura, etc.) y extrínsecas (marca, precio, imagen, etc.) que busca el consumidor en los alimentos (Fandos y Flavián, 2011), mientras que otros analizan las subjetividades (Grunert, 2006). En ese sentido, la confianza y las percepciones sobre la calidad son algunos de los elementos que diferentes autores han planteado como importantes en el análisis del consumo alimentario (Hansen, 2005a; Nguyen, Rutsaert, Van Loo y Verbeke, 2017). 


\section{PERCEPCIÓN DE LA CALIDAD Y CONFIANZA EN EL JAMÓN IBÉRICO. \\ PERSPECTIVAS DEL CONSUMIDOR Y DEL VENDEDOR \\ SÁnchez-Vega, Amaya-Corchuelo, Espinoza-OrTega}

\section{La percepción como variable subjetiva en el análisis de la calidad alimentaria}

El concepto de percepción se ha modificado con el paso del tiempo; pasó de ser lo que se aprende a través de los estímulos sensoriales, y que ha permitido a la humanidad sobrevivir y mantenerse alerta, a ser la noción de aprendizaje que crea estructuras mentales anticipadas para contrastar un estímulo recibido. Por tanto, cualquier persona evalúa una situación (objeto o sujeto) bajo factores "subjetivos y culturales" y factores "objetivos"; los primeros suelen anteponerse a los segundos y el resultado derivará en un significado (Pérez, 2012). Por consiguiente, las percepciones son un acto biocultural (Vargas, 1994) que involucra los aspectos sensoriales cuya forma de procesarlos se rige por códigos culturales aprendidos desde la infancia (Lutz, 2008) y que definen una identidad.

De acuerdo con Molano (2007), la identidad supone un reconocimiento y apropiación del pasado, en consecuencia, puede ser reconstruido o reinventado, pero al mismo tiempo debe ser reconocido y apropiado por todos los miembros del grupo cultural de referencia. Bajo estas observaciones, las percepciones son el resultado de ideas, creencias, sensaciones, motivaciones y actitudes, enmarcadas culturalmente de tal forma que no son estáticas. En ese sentido, Vargas (1994, p. 50) afirma que "las percepciones atribuyen características cualitativas a los objetos o circunstancias...lo cual permite generar evidencia de la realidad"; una de estas evidencias es lo que entendemos por calidad en los alimentos. Ahora bien ¿el término calidad es claro y objetivo?

Delimitar con precisión el concepto de calidad es arduo por la multitud de perspectivas y propuestas de análisis que existen. De forma reduccionista, una perspectiva considera que la calidad puede ser entendida como una decisión valorativa (Corfman, 1985; Zeithaml, 1988); otra que como práctica social conlleva comparar valores y atributos. Esta segunda tendencia sobre la calidad alimentaria iniciada por Olson y Jacoby (1972), pose una larga trayectoria y se basa en el análisis de atributos intrínsecos y extrínsecos. De esta forma de abordar el análisis de la calidad alimentaria, surgió uno de los enfoques más en boga, el denominado enfoque multiatributo (Aramyan, Ondersteijn, Van Kooten y Lansink, 2006) donde la multidimensinalidad de factores y la importancia del individuo en la toma de decisiones son fundamentales. 
Esta forma de entender la calidad hace referencia a los atributos distintivos de un alimento creados social e históricamente (Hernández y Villaseñor, 2014). De acuerdo con Hansen (2005a) la calidad percibida por el consumidor tiene cuatro dimensiones: a) los aspectos hedónicos, cuyo fin es el goce (Baudrilard, 2009); b) la salud, hace referencia a los aspectos nutricionales, pero también a "la naturalidad y la tradicionalidad" (Hansen, 2005b), y a la inocuidad (Lee, Lee y Lee, 2012); c) la comodidad y/o practicidad vinculada con las rutinas sociales de zonas urbanas, que se circunscriben en el tiempo limitado para la preparación de alimentos, el marketing, el aumento de la clase media, la incorporación de la mujer en el ámbito laboral, entre otros (Toffler, 1999); d) finalmente los procesos de producción, quizá el tema más debatido en los estudios alimentarios (Hansen, 2005a).

A través de los procesos se puede analizar todo el sistema agroalimentario, es decir, el sistema que opera la comida que se traslada "del campo a la mesa" en el cual intervienen además personas, organizaciones (Díaz, 2005). Teniendo esto en cuenta, Hernández y Villaseñor (2014) clasifican los sistemas agroalimentarios en dos: "actividad artesanal" donde se observa una clara cercanía del consumidor y del productor, donde lo manual y familiar predomina. Por otro lado la gran industria, es decir el "régimen alimentario corporativo" (McMichael, 2015), cuyos procesos son cuestionados por su efecto en la salud, contaminación ambiental, nutrición (Aguirre, 2004) y aspectos sociales (Carlsson-Kanyama y Linden, 2001).

El análisis de los procesos permite entender y exponer el poder de la gran industria sobre las decisiones alimentarias tanto a nivel familiar como en las políticas públicas, por ejemplo al establecer normativas de calidad sanitaria que, aunque necesarias, representan un problema para los pequeńos productores de alimentos tradicionales cuyos procesos no cumplen cabalmente las normas (Chombo-Morales, Kirchmayr, Gschaedler, Lugo-Cervantes y Villanueva-Rodríguez, 2016).

Por ello, el marco teórico del presente trabajo aborda a la calidad alimentaria como categoría de análisis vinculada fundamentalmente a aspectos culturales. El enfoque señala que estamos ante un "consumo cultural" que incluye los valores y normas de cada grupo humano en torno a la alimentación y que van más allá del mercado o la inocuidad (Portilho, 2009; Paddock, 2015; Aguilar, Amaya-Corchuelo y López, 2016). En esta esfera hay que situar los discursos sobre la percepción de la confianza y calidad de los alimentos consumidos por los participantes en la práctica alimentaria. De este modo, la calidad de las producciones agroalimentarias es 
inherente a consideraciones sociales sobre el valor ańadido, notoriedad, la singularidad del producto (Amaya-Corchuelo, Frohelich y Aguilar 2019).

Entendiendo la confianza como una parte esencial en la interrelación mercantil consustancial a la elección y adquisición de un producto en el hecho alimentario, donde entra en juego la seguridad en el vendedor que ha creado una expectativa acorde a la fiabilidad (Moorman, Deshpandé y Zaltman, 1993). Se trata de una confianza interpersonal y al tratarse de productos agroalimentarios, siempre que haya una marca entre producto y consumidor, se da la seguridad del consumidor en la capacidad de dicha marca para realizar su función correctamente (Chaudhury y Holbrook, 2001). En el primer caso la confianza se orienta hacia una persona, mientras que en el segundo se desvincula de persona alguna y se dirige hacia percepciones intangibles vinculadas a la marca (Delgado y Munuera, 2001). Finalmente se entiende que la confianza es dinámica y contextual (Solá, Prades, Espluga y Real, 2009), presenta lazos de interdependencia en la medida que no puede ser alcanzado el objetivo de uno, sin la confianza recíproca del otro (Fernández, 2013).

En síntesis, la calidad es un constructo social, que se legitima por dos vías. La primera, mediante el uso de variables objetivas y científicamente medibles, impuestas a través de instituciones con regulaciones y normativas; la segunda, a partir del reconocimiento y/o legitimación de una calidad específica por parte del consumidor, en la que se da cabida a subjetividades como son la confianza (AmayaCorchuelo et al., 2016). De ahí que el concepto de calidad no sea universal y único, pues los matices los dan los aspectos culturales, así como la confianza. Para éste trabajo, más allá del prolijo repaso del abanico de variables que determinan la calidad alimentaria, el análisis se circunscribe a las percepciones de la calidad con base en la confianza de los consumidores.

Diversos trabajos analizan la calidad de productos alimenticios centrándose en determinar las características físicas, microbiológicas y sensoriales a partir de sellos de calidad como Denominaciones de Origen, Indicaciones Geográficas, marcas colectivas, certificaciones ecológicas, comercio justo, entre otras (Chombo-Morales et al., 2016). Por parte de los productores, los sellos de calidad se han creado y proliferado como una forma de proteger su saber-hacer y sus productos (Boucher $\mathrm{y}$ Requier-Desjardins, 2005). Representa un valor añadido usado como estrategia comercial debido a que su calidad está respaldada por un bagaje cultural y por las 
estrictas normas de control que tienen que cumplir, lo cual reduce la percepción de riesgo (Fernández-Olmos y Díez-Vial, 2013).

Por otra parte, estos sellos responden al interés de los consumidores que buscan productos alternativos a los industrializados, que estén más relacionados con los sistemas tradicionales y donde exista mayor interacción ente el consumidor y el productor (Triches y Schneider, 2015); y, además, que estén exentos de escándalos alimentarios (Calle, Soler y Vara, 2012; Contreras, 2005; Nguyen et al., 2017). Ares et al. (2011) mencionan que los análisis sobre pensamientos, emociones, impresiones y asociaciones deben realizarse en un producto específico. En ese sentido el presente trabajo usará al jamón ibérico para determinar las percepciones sobre la calidad por parte de los consumidores y vendedores.

\section{Contextualizando al Jamón ibérico}

El jamón ibérico es un alimento con un saber-hacer heredado generacionalmente; su proceso está ligado al territorio denominado "Dehesa" localizado en el suroeste de la Península Ibérica. La Dehesa es un bosque antrópico, principalmente de encinas y alcornoques que producen bellotas. La montanera es el periodo de engorde extensivo de los cerdos de raza ibérica con bellota, en éste lapso se obtienen características organolépticas únicas en el cerdo. El proceso continúa con el sacrificio y despiezado del animal; el jamón corresponde a los cuartos traseros, mientras que cuartos delanteros son llamados paletas. Sigue con la curación (salado, lavado, secado y su maduración) que es un proceso crítico y largo, de hasta tres años (la norma de calidad vigente es el Real Decreto 4/2014).

Este producto emblemático de la península Ibérica, cuenta con cuatro DOP en España y una en Portugal (Aguilar et al., 2016). De manera específica, las DOP españolas han enfocado su reputación colectiva como alternativa de venta centrada en el mercado internacional (Fernández-Olmos y Díez-Vial, 2013), lo que les ha permitido posicionarse como un alimento gourmet, debido al alto precio que tienen algunos jamones; algunas páginas de internet los colocan dentro de los alimentos caros del mundo, junto con la carne Kobe, café kopi Luwak, el caviar Almas, trufa blanca, etc. (Verema, 2016). Por otra parte, se encuentran jamones ibéricos de bellota sin DOP, pero de buena calidad que son accesibles para el consumidor local. 
Es, precisamente, en este tipo de consumo donde, más allá de las marcas, el vendedor juega un papel fundamental (Amaya-Corchuelo et al., 2016).

Sobre el jamón ibérico se ha escrito un número considerable de publicaciones, destacan el análisis de los sellos de calidad (Aguilar et al., 2016), uso de nuevas tecnologías en la producción (Lorido, Estévez, Ventanas, y Ventanas, 2015), análisis de la idoneidad de los sistemas de clasificación (Marano-Marcolini y Torres-Ruiz, 2017), perfiles sensoriales con consumidores y productores (Morales et al., 2008), análisis del hedonismo, salud y sociabilidad (Amaya-Corchuelo et al., 2018), coherencia entre las preferencias hedónica y comportamiento de compra real (Resano, Sanjuán y Albisu, 2009), atributos que afectan a las preferencias (Mesías, Gaspar, Pulido, Escribano, y Pulido, 2009) e influencia del etnocentrismo (Fernández-Ferrín, Calvo-Turrientes, Bande, Artaraz-Miñón, y Galán-Ladero, 2018).

No obstante que las percepciones sobre jamón ibérico comienzan a estudiarse (Amaya-Corchuelo et al., 2016), es necesario contribuir al tema de la calidad percibida por parte de los consumidores y su relación con la construcción de la confianza. Por consiguiente, el estudio tiene como objetivo conocer las percepciones que tienen tanto el consumidor y vendedor de jamón ibérico sobre su calidad, abordando la confianza en su papel causal como facilitadora y como elemento de correlación en las experiencias personales.

\section{Metodología}

El trabajo se realizó en la ciudad española de Sevilla en los lugares de venta y consumo de jamón ibérico (supermercados, abacerías ${ }^{1}$ y mercados municipales). Debido a que fue un estudio exploratorio se siguieron los lineamientos de Ross, Clark, Padgett y Renckly (2002). La muestra estuvo conformada de 100 consumidores y 30 vendedores. Los consumidores fueron hombres y mujeres mayores de 18 ańos, que manifestaran consumir jamón y ser de la Comunidad Autónoma de Andalucía. Respecto a los vendedores, fueron encuestados en su lugar de trabajo y no se hizo distinción del tipo de establecimiento.

El cuestionario se aplicó de forma presencial. La herramienta fue sensible a la obtención de detalles culturales y sociales en torno a las percepciones del jamón 
ibérico y estuvo conformada de tres secciones: libre asociación de palabras, preguntas abiertas $y$, finalmente, los datos socioeconómicos. La primera es una técnica proyectiva que permite obtener información inconsciente o no racional como son las emociones, percepciones, actitudes (Gambaro, 2018), a través de un estímulo que puede ser una palabra o una frase (Hilverda, Jurgens y Kuttschreuter, 2016). Para este caso se usó el estímulo "buen jamón ibérico" y su asociación con una persona. Gambaro (2018) señala que este tipo de asociaciones permite descubrir valores sociales y respuestas emocionales de los consumidores hacia un producto. Las preguntas abiertas permiten dar voz al informante dentro del texto, usando literalmente algunas de sus apreciaciones culturales más significativas. Se consideraron los momentos especiales y la compañía mientras se consume jamón ibérico, la percepción que se tienen del jamón que consumen actualmente en relación con el que consumían en la infancia. De manera adicional se pidió definieran a un vendedor o al consumidor de jamón ibérico, según fuere el caso (C) consumidor $(\mathrm{V})$ vendedor.

\section{Resultados y discusión}

\section{Asociaciones}

Distintos estudios han dejado claro la importancia del sabor como uno de los criterios de compra y valoración del jamón ibérico (Mesías et al., 2009; Resano et al., 2009); así como la evidente confusión en los consumidores sobre los tipos de jamón existentes y sus distintivos. Lo anterior se profundizó a raíz de las campañas de marketing de un etiquetado desleal por parte de algunos productores y de una adaptación relativamente lenta de los consumidores a las normativas debido a los constantes cambios. Muestra de lo anterior, se observó en el presente estudio, donde ninguna persona expresó alguna asociación sobre el etiquetado. No obstante, se evidenciaron palabras bien posicionadas en el imaginario que definen un buen jamón ibérico. Las cuatro palabras más mencionadas por los consumidores fueron: bellota $(n=19)$, sabor $(n=12)$, precio $(n=11)$ y calidad $(n=8)$; por su parte los vendedores al igual que los consumidores reconocen la bellota $(n=12)$; pero 
incorporan otros aspectos como Denominación de Origen ( $n=3)$, calidad ( $n=3)$ y palabras que aluden a la identidad territorial (Andalucía, España, Sevilla).

En relación con las asociaciones del jamón ibérico con una persona, se destaca el vínculo casi en la misma proporción entre consumidores y vendedores con el género masculino (59 \% y $63 \%$ ). De acuerdo con lo descrito por los consumidores, las asociaciones se vincularon al hombre cabeza de familia, quienes además de comprarlo lo cortan: "Él es quien sabe de jamones y el que compra los mejores" (C: Mujer, 22 años) / "Es el que lo compra y lo parte" (C: Mujer, 21 años) / "Él mantiene a la familia y compra el jamón" (C: Hombre, 28 años).

Cortar un jamón es una de las cualidades que tiene alguien que conoce de jamones a tal grado que existen carreras profesionales y concursos de cortadores de jamón. Amaya-Corchuelo et al. (2018) explican que saber cortar el jamón atribuye prestigio a quien lo hace pues es resultado de un saber experto. Así lo corroboran los informantes: "Si está bien hecho, bien curado, sabe bien; hay que cuidar no estropearlo al cortarlo" (V: Hombre de 53 años) / "Que sepa lo que es el jamón, debe de saber cortarlo, empezar por un lado y cortarlo, debe ser higiénico y cortarlo fino" (C: Mujer de 49 años).

Por otro lado, las asociaciones de los consumidores vinculadas con las mujeres se relacionaron con la compra de los alimentos para el hogar y el gusto por jamón y su uso en la elaboración comida: "Trae el jamón a la casa, es la que pasa por la charcutería"- sobre la madre (C: Hombre de 23 años) / "Es la que se encarga de comprarlo" -sobre la madre (C: Mujer de 25 años) / "Ella hacía unas croquetas de jamón ibérico" -sobre la abuela (C: Hombre de 24 años).

Mientras que los vendedores relacionaron a las mujeres no con el proceso de producción sino con la transmisión del conocimiento: "De niño uno come lo que le den sus papás" (V: Hombre de 26 años) / "Mi pareja es de Extremadura y me enseñó de un buen jamón ibérico" (V: Hombre de 43 años) / "Ella (madre) me lo daba (el jamón) desde pequeña, me dio el gusto por la tradición” (V: Mujer de 54 años). Aquí se muestra que el mundo del jamón ibérico es dominado por el género masculino, sin embargo, la aparición de mujeres se ha comenzado a notar cada vez más en concursos solo de cortadoras e industrias de jamón ibérico encabezadas por mujeres.

Como se ha evidenciado con los datos anteriores, las asociaciones hechas hacia el núcleo familiar fueron las más representativas, asimismo los momentos especiales 
con quien recuerdan estar comiendo jamón ibérico concuerdan totalmente con las relaciones hechas hacia la familia nuclear y cercana en el caso de los consumidores (78 \%), a los que le secundan situaciones con amigos y colegas; mientras que los vendedores asociaron con mayor frecuencia a la familia (84 \%). Ambos grupos concuerdan en las relaciones sobre festividades familiares, por lo que estos resultados corroboran la importancia cultural y lo ya abordado por distintos autores sobre el significado familiar (Amaya-Corchuelo et al., 2018) (Tabla 1).

\section{Tabla 1.}

\section{Asociaciones emotivas del jamón ibérico}

\begin{tabular}{clcc} 
& \multicolumn{1}{c}{ Ejemplo de asociaciones } & Consumidores & Vendedores \\
Núcleo familiar & Padre, madre, esposa/o, hijos, hermanos & 59 & 67 \\
Familia cercana & Abuelo/a, tíos, suegro, sobrino, familiar & 19 & 17 \\
Externo & $\begin{array}{l}\text { Amigo, novio/a, vecino/a, andaluz, } \\
\text { compañero, compadre, jefe }\end{array}$ & 19 & 13 \\
$\begin{array}{c}\text { Mismo } \\
\text { participante }\end{array}$ & Él/ella mismo/a & 3 & 3
\end{tabular}

Fuente: elaboración propia.

Percepción de la calidad y de la confianza

Las respuestas sobre calidad, tanto de consumidores como de vendedores, permitieron identificar cuatro grupos de consumidores de acuerdo con las percepciones: que la calidad en el pasado era superior a la actual; que en la actualidad los jamones son mejores que en su infancia; que no existe diferencia aparente en la calidad, dado la tradición sigue vigente cuya forma de producción no ha cambiado; y, finalmente, están quienes muestran poco un interés sobre la calidad más allá del aspecto de sabor. Estos grupos de percepciones se nombraron de la siguiente manera: "Mejor en el pasado", "Actuales positivos", "Permanentes" y "Neutrales" (Figura 1). 


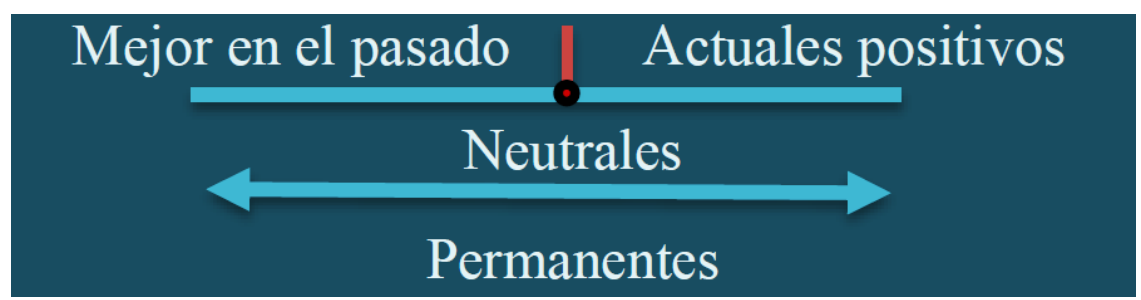

Figura 1. Grupos de percepciones.

Fuente: elaboración propia

El grupo Mejor en el pasado fue el segundo grupo más grande ( $\mathrm{n}=36)$; lo constituyeron, principalmente, personas casadas, varones de edades entre 46 a 65 ańos, con estudios de bachillerato y universitarios con ingresos bajos. El grupo Actuales positivos $(\mathrm{n}=32)$ dejan ver la confianza que tienen hacia la implementación de normativas y uso de tecnología. Se constituyó, principalmente, por mujeres de edades entre 26 a 45 ańos; con estudios universitarios e ingresos entre los 1000 y 2000 euros. Los Permanentes fueron el grupo más grande ( $\mathrm{n}=53)$, sus comentarios son alusivos a una continuidad en el consumo y sabor del jamón ibérico dado que es una tradición que no se pierde, que permanece en el tiempo. Se formó por la frecuencia mayoritaria de mujeres jóvenes ( 18 a 25 años); estudios de bachillerato y universitarios e ingresos bajos Finalmente el grupo Neutrales, fue el grupo más pequeño $(n=9)$. No percibe diferencia en la calidad en el tiempo y tampoco tiene interés en dar una explicación más amplia sobre su aseveración. Lo conformaron, principalmente, solteros con ingresos entre 500 y 1,000 euros (ver Tabla 2). 


\section{Tabla 2.}

Características socioeconómicas de los grupos consumidores de acuerdo a sus percepciones

\begin{tabular}{|c|c|c|c|c|c|}
\hline & & $\begin{array}{c}\text { Neutrales } \\
\qquad(n=9)\end{array}$ & $\begin{array}{l}\text { Mejor en el } \\
\text { pasado } \\
(\mathrm{n}=36)\end{array}$ & $\begin{array}{c}\text { Actuales } \\
\text { positivos } \\
(n=32)\end{array}$ & $\begin{array}{c}\text { Permanentes } \\
(n=53)\end{array}$ \\
\hline \multirow{4}{*}{ Edad } & De 18 a 25 & 33 & 19 & 6 & 42 \\
\hline & De 26 a 45 & 22 & 28 & 50 & 36 \\
\hline & De 46 a 65 & 44 & 47 & 41 & 23 \\
\hline & Más de 66 & 0 & 6 & 3 & 0 \\
\hline \multirow{2}{*}{ Sexo } & Hombre & 56 & 56 & 47 & 47 \\
\hline & Mujer & 44 & 44 & 53 & 53 \\
\hline & Primaria & 11 & 8 & 13 & 6 \\
\hline \multirow{3}{*}{ Estudios } & Secundaria & 22 & 22 & 19 & 17 \\
\hline & Bachillerato & 33 & 33 & 19 & 36 \\
\hline & Universitarios & 33 & 36 & 50 & 42 \\
\hline \multirow{6}{*}{ Ocupación } & Estudiante & 11 & 14 & 3 & 26 \\
\hline & Empleado & 33 & 36 & 53 & 53 \\
\hline & Independiente & 22 & 14 & 19 & 9 \\
\hline & Jubilado & 11 & 14 & 3 & 0 \\
\hline & Hogar & 11 & 6 & 9 & 2 \\
\hline & Desempleado & 11 & 17 & 13 & 9 \\
\hline \multirow{4}{*}{ Estado civil } & Soltero & 56 & 31 & 31 & 60 \\
\hline & Casado & 33 & 47 & 56 & 30 \\
\hline & Divorciado & 0 & 14 & 9 & 9 \\
\hline & Viudo & 11 & 8 & 3 & 0 \\
\hline \multirow{5}{*}{$\begin{array}{c}\text { Ingresos } \\
\text { mensuales }(€)\end{array}$} & $0-500$ & 33 & 47 & 19 & 45 \\
\hline & $501-1000$ & 11 & 17 & 22 & 28 \\
\hline & $1001-2000$ & 22 & 33 & 34 & 21 \\
\hline & Más de 2000 & 0 & 3 & 13 & 4 \\
\hline & No contestó & 33 & 0 & 13 & 2 \\
\hline
\end{tabular}

Fuente: elaboración propia.

Además de las categorías señaladas, se obtuvieron otro tipo de datos relevantes y complementarios, que refieren, fundamentalmente, a los actuales procesos de producción y la incorporación de tecnología en el sector del jamón ibérico y que permiten tener una mejor interpretación de la información. 


\section{PERCEPCIÓN DE LA CALIDAD Y CONFIANZA EN EL JAMÓN IBÉRICO.

A) Neutrales: aunque pequeño, los Neutrales son un conjunto tanto de consumidores y vendedores que no observan diferencia aparente en su percepción de la calidad del jamón con respecto a su infancia, dando respuestas ambiguas, cuyas interpretaciones más allá de una singularidad del gusto, podrían ser erróneas: "Me sabe igual, ahora consumo menos jamón, pero es por gusto" (C: Hombre de 30 ańos) "Sabe igual" (V: Hombre de 31 ańos) / "No creo que cambiara mucho el sabor" (V: Mujer de 30 años).

B) Mejor en el pasado: Kessous y Roux (2008) mencionan que la nostalgia determina las preferencias por objetos que eran habituales cuando se es más joven, además de ser un medio que coadyuva en el enfrentamiento de grandes cambios entre cada etapa de un ciclo de vida. Esto contextualiza los comentarios referidos a etapas en la juventud de las siguientes mujeres: "Antes era mejor, mis padres hacían la matanza, el jamón lo colocaban en un bidón de aceite de olivo. Yo soy de Jaén, era un buen jamón, antes se aprendía del campo, ahora la alimentación es rara, ya no es como antes, se curaba sobre la marcha, se salaba, era natural, se secaba y se metía al aceite" (C: Mujer, 88 años) / "Antes era mejor, mis abuelos hacían jamón, y era el mejor, ahora que ya no hacen ya no es el mismo" (C: Mujer, 43 años).

Las modificaciones en la producción de alimentos por la industrialización alimentaria, ${ }^{2}$ aunado al incremento en los precios como una de las problemáticas de la crisis en la producción de jamón a partir de 2007 (Chamorro, Rubio y Valero 2007), han sido causales en las nuevas formas de conceptualizar el jamón ibérico, contribuyendo a ser percibido de calidad reducida por algunos informantes: "Antes había más calidad en el producto, la comida del cochino ahora es más cara y por eso se le da de comer alimento de menor calidad, por eso ahora el cerdo es de menor calidad" (V: Hombre de 44 años) / "Antes estaba mejor, la crianza era natural, había más bellota porque no se producía tanto (cerdos) como ahora, la demanda gana" (V: Hombre de 58 años).

Esta información no es exclusiva de los ganaderos y vendedores, forma parte del conocimiento de dominio público: "Antes no había tanta cosa para darle a los animales como ahora, antes se les daban mejores cosas, más saludable y de mejor calidad" (C: Mujer de 68 años). 
En el ir creando confianza, es indispensable la adecuada comunicación de información de un tema, producto o situación (Sanzo, Santos, Vázquez y Álvarez, 2003). Estos aspectos intangibles son claves que ayudan al establecimiento de relaciones, donde el tiempo será otro elemento que ayuda a confirmar, o no, la calidad percibida (Grunert, 2006). Es decir, durante el proceso, los lazos de confianza pueden aumentar y la credibilidad en determinadas señales de calidad de un producto y serán ratificadas solo cuando se han comprobado positivamente las expectativas. Estas señales intangibles de calidad es lo que Hansen (2005a) denomina "atributos de crédito", entendidos como las propiedades del producto que solo pueden ser verificadas a partir de lo que dicen los expertos, debido a que son características que el consumidor no puede percibir y evaluar, como el saber hacer, el bienestar de los animales, los efectos a la salud a largo plazo, por mencionar algunos.

En los resultados del presente trabajo, se observa que a pesar de las normativas elaboradas en torno a la calidad de los atributos intrínsecos del jamón ibérico y de sus constantes adecuaciones, existe un desconocimiento generalizado por parte de los consumidores, por lo tanto, no todos pueden expresar un aumento en la calidad percibida con la implementación de esas normas. En ese sentido se observa una deficiencia de las instituciones en la comunicación satisfactoria de esas estrategias (Sanzo et al., 2003). Todo ello provoca que estas normativas no se contemplen como parte de los atributos de crédito, que bien podrían ser usados por los vendedores como estrategias de venta.

De manera adicional, la presión ejercida con las normas de calidad es percibida como coadyuvante aparente en la perdida de los recursos intangibles en torno a la producción de jamón: "Antes era de mejor calidad, se esforzaban más por tener un producto bueno, entre los mismos jamoneros había que mantenerse como el mejor jamonero, ahora se preocupan por cumplir las normas" (V: Mujer de 62 años) / "Ahora es de menos calidad, porque los productos que se usan para el jamón no son todos de aquí; se ha perdido el conocimiento de la curación, ahora se hace la curación industrial y por eso es de menor calidad para mí" (C: Hombre de 55 ańos).

La implementación de normativas en los procesos productivos es una de las estrategias empleadas en la generación de calidad institucional, lo que brinda una ventaja competitiva; a su vez, el uso de distintivos que amparan cierta calidad intrínseca y extrínseca, como son las Denominaciones de Origen, son elementos que 
han demostrado éxito en el mercado, en parte debido a los márgenes de ganancias. Por otro lado, estos distintivos son excluyentes no solo para quienes puedan gestionar y llevar a cabo producciones con ciertas características, sino que son exclusivas para quien pueda pagarlas: "Ahora se vende para más gente, y la ley de la oferta y la demanda lo marca; se come menos del bueno; del bueno, bueno solo lo comen los ricos, el jamón ahora es un lujo" (C: Mujer de 57 años).

C) Actuales positivos: la incertidumbre que ha generado la industrialización de la comida a partir de escándalos alimentarios y la desconexión entre productores y consumidores en las cadenas productivas, contribuyó a que algunos consumidores comenzaran a exigir garantías mediante normativas en el etiquetado en los alimentos, que dieran fe de las formas de producción y de los insumos empleados. A pesar de ello existe la llamada "agro-mafia" (término italiano que se extendió del fraude en el aceite de oliva etiquetado como "hecho en Italia") para referir el fraude alimentario en general en el etiquetado.

En el mundo del jamón ibérico abunda la picaresca (habilidad de engañar) tanto en la producción como en la distribución. Fernández-Zarza et al. (2018) reportan que del total de los cerdos sacrificados del 2012 al 2014 solo el $5.5 \%$ pertenecía a la raza ibérica pura, sin embargo, había una mayor proporción en el mercado de producto etiquetado como ibérico de bellota. Este hecho fue uno de los determinantes para la implementación de etiquetado con precintos de colores que ayudaran al consumidor a reconocer la pureza del animal y el tipo de alimentación con que ha sido producido.

Ante esto, es posible que las percepciones que los consumidores tienen sobre el jamón se deban, además del marketing, a la efectividad en el tipo de comunicación empleada por los facilitadores, entendidos como los "marcos institucionales derivados de políticas y regulaciones preexistentes, dirigidos por grupos de poder corporativos, garantizados y respaldados por la administración tanto a nivel gerencial y financiera" (Fernández-Zarza et al., 2018, p. 14). Estos marcos institucionales, ayudan a que las recomendaciones que hacen los vendedores sobre algún producto tengan una base confiable, lo cual se corrobora con los discursos que manejan sobre la calidad del jamón: "Ahora es mejor, con la DO se cumplen estándares y es mejor" (V: Mujer de 56 años) / "Ahora hay más normas que hay que seguir y eso lo hace mejor" (V: Hombre de 43 años). 
Al grupo Actuales positivos de manera contraria al grupo Mejor en el pasado, los cambios en las formas de producir alimentos con el uso de tecnologías, la aplicación de normativas en la producción, el empleo de alimentos industrializados para los animales, los avances científicos en la conservación de alimentos, etc., les generan tranquilidad: "Es mejor ahora, la tecnología y alimentación de los cerdos es ahora mejor, antes los cerdos comían desperdicios" (C: Hombre de 65 años) / "Ahora es mejor, hay más regulaciones" (C: Mujer de 61 años) / "Ahora es mejor, con la alimentación del cochino y más tecnología es mejor (el jamón) "(C: Hombre de 41 ańos) / "Ahora es mejor, ahora como consumidores somos más exigentes con la calidad y hacen eso" (C: Mujer de 35 años).

Chamorro et al. (2007) mencionan que dentro de los factores que determinan la compra de un jamón está la procedencia, el precio y en menor medida lo que le dice el vendedor, es decir, las recomendaciones. Pero en el presente trabajo de acuerdo con las concepciones de los consumidores sobre los vendedores de jamón se observa lo contrario, y coincide con la investigación de Fandos (2016) sobre el jamón de Teruel, donde demuestra que cuando el vendedor no recomendó el jamón se afectó la decisión de compra de los consumidores. Dicho de otro modo, dentro de los aspectos que se toman en cuenta en la compra destacan las recomendaciones y la percepción que tienen del vendedor: "Una persona correcta, que explica bien, no engaña, que sea franco, y amable" (C: Mujer de 68 años) / "Persona sincera y de confianza del producto que vende, no un charlatán de feria que vende lo malo, lo más importante es la confianza" (C: Mujer de 57 ańos) / "Un profesional bueno, bueno, que sea bueno y se exprese bien, que venda cosas buenas y no diga que es bueno cuando no es asî" (C: Hombre de 45 años).

Ante estos comentarios también se puede concluir que al romperse la cercanía entre productor y consumidor, la percepción y la confianza depositadas en el vendedor son seńales que inferirán como parte de la calidad, que al comerse el producto será ratificada o no: "Alguien que sabe engañar bien, vende diciendo que es algo y es otra calidad, aprovecha que no sabemos" (C: Mujer de 21 años) / "En otra época había más falta de alimentos por eso no se consumía jamones caros, ahora es más fácil adquirir un buen jamón con menos dinero, antes no todos comían jamón" (C: Hombre de 42 años).

De acuerdo con la citada autora, la confianza es un aspecto fundamental que permite incorporar características altamente valoradas por los consumidores en los 
productos alimenticios (higiene, valor nutricional, entre otros) lo que disminuye el riesgo percibo; además cuando no se tiene una cantidad suficiente de información se recurrirá a la confianza en el vendedor. No obstante, los vendedores hacen uso de las normativas en sus discursos para justificar efectivamente la calidad, lo que muestra el grado de implicación entre los agentes y el producto: "Es diferente ahora, mejor, ahora está la DO que garantiza la calidad, pero, aunque antes no había no era malo, también era bueno "(V: Mujer de 55 ańos) / "Ahora las normativas avalan la calidad, pero antes, aunque no había estas normas tal como ahora se consumía buen jamón, y con la tecnología es todo distinto, se controla mejor la curación” ( $\mathrm{V}$ : Mujer de 47 años).

Mediante estos últimos datos se comprueba una calidad que, aunque es mejor percibida en la actualidad debido a las normativas y avances en la tecnología, no es exclusiva de ahora, sino de tiempo atrás con el saber hacer generado y transmitido generacionalmente.

D) Permanentes: diversos trabajos que estudian los alimentos tradicionales tienen en común el vínculo con la identidad, la aportación nutricional y la frecuencia de consumo, puesto que muchos son de primera necesidad. Sin embargo, AmayaCorchuelo et al. (2018) mencionan que a pesar de que el jamón es un producto tradicional su frecuencia de consumo no depende exclusivamente de la identidad sino del poder adquisitivo, ya que es un producto de lujo no esencial. En ese sentido resulta interesante observar la profundidad identitaria del jamón ibérico en el imaginario de la muestra de estudio.

Las conceptualizaciones hechas por los vendedores sobre las características que tienen los consumidores de jamón ibérico son referidas a su poder adquisitivo (tiene clase, es de estatus alto) y a su capacidad para degustar lo mejor, lo delicatessen. En otras palabras, los consumidores son conceptualizados a partir de un aspecto hedónico, lo cual guarda relación con lo dicho por Neme y Rodríguez-González (2013) sobre el papel de un producto como elemento posicional en un grupo social, ya que no todos pueden poseerlo. De manera adicional se les reconoce como conocedores informados del buen comer y, en menor medida, como partidarios de continuar con la tradición de consumo de un buen jamón ibérico.

Espeitx (1996) menciona que los productos tradicionales existen en la medida en que permanecen en el tiempo y se producen en cierto lugar y de cierta manera, además de ser concebidos como naturales. Esto sucede en el caso que nos ocupa: 
"La tradición de curar jamones no cambia" (V: Mujer de 34 ańos) / "De lo que depende es del jamón que compras, si es de mejor calidad o no" (V: Hombre de 32 años) / "Soy del campo y ahí hacemos nuestro propio jamón, siempre como el jamón que nosotros producimos" (C: Mujer de 18 años) / "Porque es una tradición que siempre se ha mantenido" (C: Hombre de 24 años).

Para que un producto sea considerado importante en el imaginario colectivo, se requiere compartir un estilo de vida y estructura de valores. En virtud de ello, los momentos especiales que hicieron mención los consumidores y vendedores están relacionados con festividades y rituales importantes (sobre todo del ciclo de vida) como bodas, bautizos, navidades, ferias, por citar algunos. De modo que aquí se corrobora por un lado lo mencionado por otros autores sobre las circunstancias en las que se consume jamón ibérico (Amaya-Corchuelo et al., 2018), y por otro, lo expresado por Guerrero et al. (2010) sobre la relación de los productos tradicionales con situaciones familiares y la práctica en ocasiones especiales.

En ese tenor y de acuerdo con Fandos y Flavián (2011), la familiaridad entendida como el nivel de entendimiento de un producto y el grado de experiencia sobre el mismo, son características indispensables para que el consumidor establezca lazos de confianza con una marca, producto y/o servicio, debido a su inferencia con una menor percepción del riesgo. En este sentido, un incremento de la confianza permite la creación de relaciones con las mismas marcas de consumo habitual, con personas y situaciones: "Cuando se da con algo bueno jamás se cambia" (C: Hombre de 28 ańos) / "Uno sigue la tradición de comprar el buen jamón que compraban nuestros padres, si era bueno, sigues comprando del bueno" (C: Mujer de 29 ańos) / "Debe explicar bien lo que vende, y hacer confianza" (C: Mujer, 35 años).

Estas interpretaciones muestran la importancia de lo que Fernández-Olmos y Díez-Vial (2013) llaman "conocimiento tecnológico no codificable", que asociaron con los conocimientos de los recursos humanos en las pequeńas y medianas empresas de jamón ibérico; sin embargo, en este trabajo bien puede observarse cómo los consumidores le atribuyen este "conocimiento" a los vendedores, al otorgarles prestigio como conocedores o expertos cualificados y entendidos de la cultura del jamón ibérico, lo que se convierte en una ventaja competitiva.

Adicionalmente, se observa a la cercanía entre actores de este ámbito cultural (suroeste de la Península Ibérica) como elemento clave del contexto que ayuda en la generación de lazos de confianza. El grado de implicación que el consumidor percibe 
en el vendedor reduce el riesgo percibido, lo que se transformará en confianza: "Debe ser un conocedor de la cultura del ibérico, lo lleva en la sangre" (C: Hombre de 41 años) /"Alguien relacionado con el jamón, que sabe bien, está enterado (C: Hombre de 62 años) / "Alguien que sabe vender el producto, que sabe de lo relacionado con el jamón, su crianza" (C: Hombre de 52 ańos) / "Es una persona que lleva el jamón en sí, que se ha criado con la cultura del cerdo" (C: Hombre de 23 años) / "Debe ser agradable, que sepa entender al jamón y al consumidor, amable (C: Mujer de 55 años).

De acuerdo con Grunert (2007), la calidad percibida es una expresión del estilo de vida. En ese sentido el grado de experiencia y el conocimiento (familiaridad) que se tiene (Fandos y Flavián, 2011) sobre el jamón ibérico, serán elementos contextuales en la generación de confianza entre actores que permita que los atributos de crédito sean elementos percibidos efectivamente como parte de la calidad extrínseca del jamón. La modernidad alimentaria ha evolucionado haciéndose sumamente compleja por lo que el consumidor ha generado un conocimiento parcial de ella (Contreras, 2005), tal como en el contexto descrito en el jamón ibérico, donde la credibilidad y la confianza juegan un papel importante en el proceso de consumo (Becker, 2000).

El vendedor es un agente clave en el proceso e incide en la disminución de la incertidumbre mediante la creación de lazos de confianza, con el establecimiento de una relación de intercambio y/o cooperación, la cual está fundamentada en las percepciones, competencias, honestidad, franqueza y compromiso. El tipo de intercambio está íntimamente relacionado con la vulnerabilidad percibida, que se justifica en las experiencias y nexos pasados, además de la información que se posee (Fernández, 2013). Sobre éste último punto el funcionamiento de las instituciones tiene un papel importante en la creación de percepciones y la aceptabilidad de ciertos riesgos, mediante su capacidad para establecer normativas y hacerlas cumplir (Fernández-Zarza, Amaya-Corchuelo, y Aguilar, 2018). En ese sentido, la confianza puede estar generada además por la proximidad local (Fonte, 2008) y la pertenencia de dichos actores de la cadena al mismo ámbito cultural donde se produce y consume el producto (Aguilar et al., 2016). Siegrist, Visschers y Hartmann (2015) explican que hay una menor percepción de riesgo en la medida en que existe mayor conocimiento sobre el producto y mayor confianza, tal como se observó en el presente trabajo. 
A través de los datos obtenidos y su análisis, se demuestra que el vendedor es una parte esencial de la cadena del jamón ibérico en la comunicación con el consumidor y su formación de percepciones sobre la calidad. Sin embargo, los resultados muestran dos limitaciones, la primera es que no se hizo una clasificación del tipo de vendedor, y la segunda que el tamaño de la muestra no permite hacer generalizaciones, por lo cual para futuras investigaciones será necesario atender estas sugerencias que ayudarán a establecer patrones de consumo con pautas contextuales específicas en la construcción de lazos de confianza.

Finalmente, las respuestas que mostraron circunstancias particulares con recuerdos de infancia invitan a plantearse el análisis de la nostalgia de una forma más profunda como variable de estudio sobre el apego emocional hacia el jamón ibérico de bellota.

\section{Conclusiones}

Como se demuestra en este trabajo la importancia del estudio de las percepciones alimentarias es fundamental para comprender el hecho alimentario en nuestras sociedades. En dicho contexto, la apreciación sobre la calidad puede ser abordada desde su subjetividad mediante los conceptos de percepción y confianza, las cuales constituyen dos variables que, mediante las prácticas sociales en torno a la alimentación, son legitimadas por los agentes de la cadena alimentaria. En ese sentido, este trabajo evidencia cómo en la calidad percibida del jamón ibérico se construye en parte por los lazos de confianza entre consumidor y vendedor.

Por otro lado, existe una generalización en la asociación de la calidad con la tradición de producción y consumo de jamón ibérico, y con la zona de origen. Se muestra además que de las preocupaciones de los consumidores sobre la calidad del jamón ibérico, el aspecto de la naturalidad en la alimentación de los cerdos es un elemento importante para el grupo llamado Mejor en el pasado; no se hizo evidente la inquietud hacia al tipo de producto terminado, es decir, si el jamón adquirido era de cebo (alimentado sólo con concentrados), recebo (uso parcial de concentrados), cien por ciento ibérico o no (el tipo de raza), sino a una preocupación sobre las características de los insumos industriales empleados en la alimentación del ganado. Todo ello indica la sospecha del consumidor hacia el resto de los procesos en la 
cadena y por consiguiente en el producto final, al percibir la alimentación del cerdo como de menor calidad se asume que el producto terminado tendrá la misma condición. De esta manera, la confianza hacia el vendedor crece en la medida en que se le percibe como experto y /o conocedor del contexto y del producto, donde son fundamentales las experiencias positivas que refuerzan la rutina de comprar al vendedor habitual con quien están familiarizados.

La percepción de un comprador sobre los atributos de crédito del jamón que ha adquirido tiene su justificación en la confianza. Los lazos de confianza entre el consumidor y vendedor se observan mediante las respuestas positivas o negativas, en función del contexto y experiencias pasadas. Asimismo, existe una conexión con el nivel de confianza hacia el vendedor (Actuales positivos), en tanto que los consumidores perciben que los facilitadores establecen y hacen cumplir las normativas que rigen la producción y distribución del jamón ibérico.

Por otra parte, la información del producto es siempre inherente al contexto cultural y la socialización recibida por el consumidor; por tanto, se afirma que se da una fuerte relación entre percepciones, selección alimentaria y confianza con el contexto (sobre todo local) donde el consumidor ha generado sus experiencias vitales.

Notas al pie:

1 Lugar de venta al por menor de distintos productos alimenticios como legumbres, aceites, vinos, y distintos productos cárnicos, donde se destacan los derivados del cerdo ibérico, entre ellos el jamón. En algunos de estos sitios también hay venta de tapas (pequeños aperitivos) y bebidas alcohólicas que se consumen en el mismo lugar.

2 La industrialización alimentaria no excluye los alimentos empleados para la cría y engorde de los cerdos, como son piensos y concentrados industriales usados en el cebo intensivo o extensivo (Vargas y Aparicio, 2001). 


\section{Bibliografía}

Aguilar, E., Amaya-Corchuelo, S. y López, I. (2016). Alimentos con calidad. Nuevas estrategias rurales para nuevos consumidores. Arxiu d'Etnografia de Catalunya, (16), 137-152. https://doi.org/10.17345/aec2016137-152

Aguirre, P. (2004). Ricos flacos y gordos pobres. La alimentación en crisis (3a ed.). Buenos Aires: Capital Intelectual. https://doi.org/10.1017/CBO9781107415324.004

Amaya-Corchuelo, S., Fernandez-Zarza, M. y Aguilar, E. (2018). Placer, salud y sociabilidad. El hecho alimentario a través del jamón ibérico. Dialectología y Tradiciones Populares, 73(2), 425-452. https://doi.org/https://doi.org/10.3989/rdtp. 2018.02.008

Amaya-Corchuelo, S., Froehlich, J. y Aguilar, E. (2019). Singularidades en venta. Uso de valores culturales y construcción de la distintividad en los casos de jamon ibérico en España y de la carne de la Pampa en Brasil. AGER: Revista de Estudios sobre Despoblación y Desarrollo Rural, 26, 35-66. https://doi.org/10.4422/ager.2018.10

Amaya-Corchuelo, S., Sánchez-Vega, L., Aguilar Criado, E. y Espinoza-Ortega, A. (2016). Percepción del consumo del consumo de producciones de calidad. Los casos del quesillo de Oaxaca, México, y el jamón ibérico de bellota de España. En M. C. Renard Hubert (Ed.), Mercados y desarrollo local sustentable (pp. 31-48). México: Red Sial-México, Conacyt, Colofón.

Aramyan, L., Ondersteijn, C., Van Kooten, O. y Lansink, A., (2006). Performance Indicators in Agri-food Production chains. En Ondersteijn, J., Wijnands, J., Huirne R., y Kooten, O. (Ed), Quantifying the agri-food supply chain (pp. 47-64). Netherlands: Springer.

Ares, G., Piqueras-Fiszman, B., Varela, P., Morant Marco, R., López, A. M. y Fiszman, S. (2011). Food labels: Do consumers perceive what semiotics want to convey? Food Quality and Preference, 22(7), 689-698. https://doi.org/10.1016/j.foodqual.2011.05. 006

Baudrilard, J. (2009). La sociología de consumo: sus mitos, sus estructuras (2a ed.). Madrid: Siglo XXI de España Editores.

Becker, T. (2000). Consumer perception of fresh meat quality: a framework for analysis. British Food Journal, 102(3), 158-176. https://doi.org/https://doi.org/10.1108/00070 700010371707

Belletti, N., Garriga, M., Aymerich, T. y Bover-Cid, S. (2013). Inactivation of Serratia liquefaciens on dry-cured ham by high pressure processing. Food Microbiology, 35(1), 34-37. https://doi.org/10.1016/j.fm.2013.03.001 


\section{PERCEPCIÓN DE LA CALIDAD Y CONFIANZA EN EL JAMÓN IBÉRICO. \\ PERSPECTIVAS DEL CONSUMIDOR Y DEL VENDEDOR \\ SÁNCHEZ-Vega, AMAYA-Corchuelo, ESPINOZA-OrTEGA}

Boucher, F. y Requier-Desjardins, D. (2005). La concentración de las queserías rurales de Cajamarca: retos y dificultades de una estrategia colectiva de activación. Agroalimentaria, 11(21), 13-27.

Calle, A., Soler, M. y Vara, I. (2012). La desafección al sistema agroalimentario: ciudadanía y redes sociales. Interface Journal, 4(22), 459-489.

Carlsson-Kanyama, A. y Linden, A. L. (2001). Trends in food production and consumption: Swedish experiences from environmental and cultural impacts. International Journal of Sustainable Development, 4(4), 392. https://doi.org/ 10.1504/IJSD.2001.001558

Chamorro, A., Rubio, S., y Valero, V. (2007). El jamón ibérico: análisis de la industria y del consumidor extremeño. En La agricultura y la ganadería extremeña en 2007 (pp. 247-260).

Chaudhury, A. y Holbrook, M. (2001). The chain of effects from brand trust and brand affect to brand performance: the role of brand loyalty. Journal of Marketing, 65(2), 8193.

Chombo-Morales, P., Kirchmayr, M., Gschaedler, A., Lugo-Cervantes, E. y VillanuevaRodríguez, S. (2016). Effects of controlling ripening conditions on the dynamics of the native microbial population of Mexican artisanal Cotija cheese assessed by PCRDGGE. LWT - Food Science and Technology, 65, 1153-1161. https://doi.org/10.1016/ j.lwt.2015.09.044

Contreras, J. (2005). La modernidad alimentaria: entre la sobreabundancia y la inseguridad. Revista Internacional de Sociologia, 63(40), 109-132. https://doi.org/ 10.3989/ris.2005.i40.191

Delgado, E. y Munuera, J. (2001). Brand trust in the context of consumer loyalty. European Journal of Marketing, 35(11), 1238-1258.

Díaz, C. (2005). La modernidad alimentaria. Los debates actuales en la sociología de la alimentación. Revista Internacional de Sociología, 40(enero-abril), 47-78.

Escobar-López, S. Y., Espinoza-Ortega, A., Vizcarra-Bordi, I. y Thomé-Ortiz, H. (2017). The consumer of food products in organic markets of central Mexico. British Food Journal, 119(3), 558-574. https://doi.org/10.1108/BFJ-07-2016-0321

Espeitx, E. (1996). Los nuevos consumidores o las nuevas relaciones entre el campo y la ciudad a través de los productos de la tierra. Agricultura y Sociedad, (80-81), 83-116.

Espinoza-Ortega, A., Thomé-Ortiz, H., Martínez-García, C. G. y Vizcarra-Bordi, I. (2016). Motives for food choice of consumers in Central México. British Food Journal, 118(11), 2744-2760. https://doi.org/10.1108/09574090910954864 
Fandos, C. (2016). Exploring the mediating role of trust in food products with protected designation of origin. The case of "jamón de teruel”. Spanish Journal of Agricultural Research, 14(1), 1-10. https://doi.org/10.5424/sjar/2016141-7169

Fandos, C. y Flavián, C. (2011). Las respuestas del consumidor ante la calidad percibida: una propuesta para productos agroalimentarios de calidad. Spanish Journal of Rural Development, 2(1), 37-52. https://doi.org/10.5261/2011.GEN1.04

Fernández-Ferrín, P., Calvo-Turrientes, A., Bande, B., Artaraz-Miñón, M., y GalánLadero, M. M. (2018). The valuation and purchase of food products that combine local, regional and traditional features: The influence of consumer ethnocentrism. Food Quality and Preference, 64(February), 138-147. https://doi.org/10.1016/j. foodqual. 2017.09.015

Fernández-Olmos, M. y Díez-Vial, I. (2013). Effect of firm’s resources on international diversification: An application in the Iberian Ham industry. European Management Journal, 31(2), 196-208. https://doi.org/10.1016/j.emj.2012.04.001

Fernández-Zarza, M., Amaya-Corchuelo, S. y Aguilar-Criado, E. (2018). Institutional density and public policies in two cases of geographical indications from Mexico and Spain. Journal of Agrarian Change, 1-19. https://doi.org/10.1111/joac.12276

Fernández, M. C. (2013). La confianza como instrumento de análisis en la cadena de valor alimentaria. Madrid: Universidad Politécnica de Madrid.

Fonte, M. (2008). Knowledge, food and place. A way of producing, a way of knowing. Sociologia Ruralis, 48(3), 200-222. https://doi.org/10.1111/j.1467-9523.2008. 00462.x

Gambaro, A. (2018). Projective techniques to study consumer perception of food. Current Opinion in Food Science, 21, 46-50. https://doi.org/10.1016/j.cofs.2018. 05.004

Grunert, K. G. (2006). How consumers perceive food quality. En L. Frewer y H. Van Trijp (Eds.), Understanding Consumers of Food Products (1a ed., pp. 181-199). Estados Unidos: Woodhead Publishing.

Guerrero, L., Claret, A., Verbeke, W., Enderli, G., Zakowska-Biemans, S., Vanhonacker, F., Issanchou, S., Sajdakowska, M., Granli, B., Scalvedi, L. Contel, M. y Hersleth, M. (2010). Perception of traditional food products in six European regions using free word association. Food Quality and Preference, 21(2), 225-233. https://doi.org/ 10.1016/j.foodqual.2009.06.003

Hansen, T. (2005a). Rethinking consumer perception of food quality. Journal of Food Products Marketing, 11(2), 75-93. https://doi.org/10.1300/J038v11n02

Hansen, T. (2005b). Understanding consumer perception of food quality: The cases of shrimps and cheese. British Food Journal, 107(7), 500-525. https://doi.org/ $10.1108 / 00070700510606909$ 


\section{PERCEPCIÓN DE LA CALIDAD Y CONFIANZA EN EL JAMÓN IBÉRICO. \\ PERSPECTIVAS DEL CONSUMIDOR Y DEL VENDEDOR \\ SÁNCHEZ-Vega, AMAYA-Corchuelo, Espinoza-OrTEga}

Hernández, M. D. C. y Villaseñor, A. (2014). La calidad en el sistema agroalimentario globalizado. Revista mexicana de sociología, 76(4), 557-582.

Hilverda, F., Jurgens, M. y Kuttschreuter, M. (2016). Word associations with "organic": What consumer think of? British Food Journal, 118(12), 2931-2948. https://doi.org/10.1108/BFJ-05-2016-0229

Jaramillo, J. L. (2015). Preferencias del consumidor y disposición a pagar por el consumo de tortilla de maíz orgánico. Estudios Sociales, 25(47), 145-161.

Kessous, A. y Roux, E. (2008). A semiotic analysis of nostalgia as a connection to the past. Qualitative Market Research: An International Journal, 11(2), 192-212. https://doi.org/10.1108/13522750810864440

Lee, G. I., Lee, H. M. y Lee, C. H. (2012). Food safety issues in industrialization of traditional Korean foods. Food Control, 24(1-2), 1-5. https://doi.org/10.1016/ j.foodcont.2011.09.014

Lorido, L., Estévez, M., Ventanas, J., y Ventanas, S. (2015). Comparative study between Serrano and Iberian dry-cured hams in relation to the application of high hydrostatic pressure and temporal sensory perceptions. LWT - Food Science and Technology, 64(2), 1234-1242. https://doi.org/10.1016/j.lwt.2015.07.029

Lutz, B. (2008). Los sentidos de la existencia ordinaria: de la percepción a la alimentación. Argumentos, 21(57), 213-218.

Marano-Marcolini, C. y Torres-Ruiz, F. J. (2017). A consumer-oriented model for analysing the suitability of food classification systems. Food Policy, 69(136), 176-189. https://doi.org/10.1016/j.foodpol.2017.04.004

McMichael, P. (2015). Regímenes alimentarios y cuestiones agrarias. México: MA Porrua.

Mesías, F. J., Gaspar, P., Pulido, Á. F., Escribano, M. y Pulido, F. (2009). Consumers' preferences for Iberian dry-cured ham and the influence of mast feeding: An application of conjoint analysis in Spain. Meat Science, 83(4), 684-690. https://doi.org/10.1016/j.meatsci.2009.08.004

Moorman, C., Deshpandé, R., y Zaltman, G. (1993). Factors affecting trust in market research relationships. Journal of Marketing, 57(1), 81-101.

Morales, R., Guerrero, L., Claret, A., Guàrdia, M. D. y Gou, P. (2008). Beliefs and attitudes of butchers and consumers towards dry-cured ham. Meat Science, 80(4), 10051012. https://doi.org/10.1016/j.meatsci.2008.04.015

Neme, S. R. y Rodríguez-González, L. (2013). Consumo simbólico: una perspectiva sociocultural en la comprensión del comportamiento del consumidor. Revista Iberoamericana de Psicología: Ciencia Y Tecnología, 6(2), 27-33. 
Nguyen, H. D. M., Rutsaert, P., Van Loo, E. J. y Verbeke, W. (2017). Consumers' familiarity with and attitudes towards food quality certifications for rice and vegetables in Vietnam. Food Control, 82, 74-82. https://doi.org/10.1016/j.foodcont. 2017.06.011

Olson, J. y Jacoby, J. (1972). Cue utilization in the quality perception process. En: M. Venkatesan (Ed.) Proceedings of the Third Annual Conference of the Association for Consumer Research, (pp. 167-179) Chicago: Association for Consumer Research.

Paddock, J. (2015). Invoking simplicity: "Alternative" food and the reinvention of distinction. Sociología Ruralis, 55(1), 22-40.

Pérez, Ó. (2012). Algunos porqués cognitivos del análisis semiótico: una aproximación a las confluencias entre Semiótica y Psicología Cognitiva. Zer: Revista de Estudios de Comunicacion, 17(33), 101-117.

Portilho, F. (2009). Novos atores no mercado: movimentos sociais econômicos e consumidores politizados. Politica \& Sociedade, 8(15), 199-224.

Resano, H., Sanjuán, A. I., y Albisu, L. M. (2009). Consumers' acceptability and actual choice. An exploratory research on cured ham in Spain. Food Quality and Preference, 20(5), 391-398. https://doi.org/10.1016/j.foodqual.2009.03.002

Ross, K. C., Clark, L. D., Padgett, T. C. y Renckly, T. R. (2002). Air University sampling and surveying handbook: Guidelines for planning, organizing, and conducting surveys. Washington DC: University Press of the Pacific.

Sánchez, M. y Barrena, R. (2004). El consumidor ante los alimentos de nueva generación: alimentos funcionales y alimentos transgénicos. Revista Española de Estudios Agrosociales y Pesqueros, (204), 95-127.

Sanzo, M. J., Santos, M. L., Vázquez, R. y Álvarez, L. I. (2003). The effect of market orientation on buyer-seller relationship satisfaction. Industrial Marketing Management, 32(4), 327-345. https://doi.org/10.1016/S0019-8501(01)00200-0

Siegrist, M., Visschers, V. H. M., y Hartmann, C. (2015). Factors influencing changes in sustainability perception of various food behaviors: Results of a longitudinal study. Food Quality and Preference, 46, 33-39. https://doi.org/10.1016/j.foodqual.2015.07.006

Solá, R., Prades, A., Espluga, J. y Real, M. (2009). Confianza, incertidumbre y percepción social de las tecnologías avanzadas. Un estudio de caso. Revista Internacional de Sociología, 67(1), 161-175.

Toffler, A. (1999). La tercera ola (16a ed.). Bogotá, Colombia: Plaza y Janes.

Triches, R. M. y Schneider, S. (2015). Alimentação, sistema agroalimentar e os consumidores: novas conexôes para o desenvolvimento rural. Cuadernos de Desarrollo Rural, 12(75), 55-75. https://doi.org/10.11144/Javeriana.cdr12-75.asac 


\section{PERCEPCIÓN DE LA CALIDAD Y CONFIANZA EN EL JAMÓN IBÉRICO. \\ PERSPECTIVAS DEL CONSUMIDOR Y DEL VENDEDOR \\ SÁNCHEZ-VEGA, AMAYA-Corchuelo, Espinoza-OrTEga}

Vargas, J. de D. y Aparicio, M. A. (2001). Análisis de la evolución de los censos y sistemas de producción del cerdo ibérico. Revista Española de Estudios Agrosociales y Pesqueros, 193, 87-118.

Vargas Melgarejo, L. M. (1994). Sobre el concepto de percepción. Alteridades, 4(8), 4753.

Zeithaml, V. (1988). Consumer perceptions of price, quality, and value: a means-end model and synthesis of evidence. Journal of Marketing, 52(3), 2-22. 